\title{
Self-adjusted convex approximation method for structural optimization
}

\author{
Tien-Tung Chung *, Chyou-Huey Chiou \\ Department of Mechanical Engineering, National Taiwan University, Taipei 10617, Taiwan, ROC \\ Received 12 November 1998; accepted 19 April 2000
}

\begin{abstract}
This study presents an approximation method called self-adjusted convex approximation for optimum design of structures. The proposed method is a generalization of the convex approximation method. The order of the approximation for each constraint is automatically adjusted in the optimization process. This self-adjusted capability makes the approximate constraint values conservative enough to maintain the optimum point of the approximate problem in the feasible region. This feature can prevent oscillation of the optimum design point and increase the convergence rate of the redesign process. Results obtained from three examples demonstrate the effectiveness of the proposed method. (c) 2001 Elsevier Science Ltd. All rights reserved.
\end{abstract}

Keywords: Convex approximation; Structural optimization; Approximation method

\section{Introduction}

The structural optimization problem considered herein consists of minimizing an objective function subject to constraints that ensure the feasibility of the structural design. The numerical optimization problem can be written in the following form:

minimize $F(\vec{x})$

subject to $h_{q}(\vec{x}) \leqslant 0, q=1,2, \ldots, n_{\mathrm{c}}$,

where $\vec{x}$ denotes the design vector, $x_{i}$ represents the $i$ th component of the design vector, $F(\vec{x})$ is the objective function, $h_{q}(\vec{x})$ denotes the $q$ th constraint and $n_{\mathrm{c}}$ represents the total number of constraints. In general, design constraints include behavior constraints and size constraints (e.g. move limits). Behavior constraints generally impose limitations on structural response quantities (e.g. placing upper bounds on stresses and displacements under static loading cases or lower bounds on natural frequencies).
As is well known, structural optimization is severely restricted by the high computational cost of solving large-scale problems. Many innovative approaches have been proposed in the recent decade to resolve this design optimization problem. Among these, the most widely used approach applies an approximation concept that replaces the original optimization problem with a sequence of explicit approximate sub-problems. This method generates the sub-problems through Taylor series expansion of the objective function and constraints in terms of intermediate design variables.

\section{Approximation methods}

\subsection{Linear approximation}

The most commonly used approximation is the firstorder Taylor series expansion [1] in terms of the original design variable. It can be written in the following form:

$$
h_{\mathrm{d}}(\vec{x})=h\left(\vec{x}_{0}\right)+\sum_{i=1}^{n}\left(\frac{\partial h}{\partial x_{i}}\right)_{\vec{x}_{0}}\left(x_{i}-x_{i 0}\right)
$$

\footnotetext{
${ }^{*}$ Corresponding author.
} 
where $h_{\mathrm{d}}(\vec{x})$ denotes the direct linear approximation function of $h(\vec{x})$ and $\left(\partial h / \partial x_{i}\right)_{\vec{x}_{0}}$ represents the sensitivity of $h(\vec{x})$ with respect to the $i$ th design variable at the original design $\vec{x}_{0}$. For some applications, the linear approximation is inaccurate even when the design point $\vec{x}$ is close to $\vec{x}_{0}$.

\subsection{Reciprocal approximation}

The reciprocal approximation is the first-order Taylor series expansion in terms of the reciprocals $1 / x_{i}[2,3]$. It can be expressed as

$h_{\mathrm{r}}(\vec{x})=h\left(\vec{x}_{0}\right)+\sum_{i=1}^{n}\left(\frac{\partial h}{\partial x_{i}}\right)_{\vec{x}_{0}}\left(x_{i}-x_{i 0}\right)\left(\frac{x_{i 0}}{x_{i}}\right)$,

where $h_{\mathrm{r}}(\vec{x})$ is the reciprocal approximation of $h(\vec{x})$. Its scaling property makes this approximation more efficient for structures with stress and displacement constraints than the appropriate linear approximation does.

\subsection{Convex approximation}

The convex approximation, generally referred to as convex linearization (CONLIN) [4], is a hybrid of the linear and reciprocal approximations. It can be written in the following form:

$$
\begin{aligned}
h_{\mathrm{c}}(\vec{x})= & h\left(\vec{x}_{0}\right)+\sum_{i}^{(+)}\left(\frac{\partial h}{\partial x_{i}}\right)_{\vec{x}_{0}}\left(x_{i}-x_{i 0}\right) \\
& +\sum_{i}^{(-)}\left(\frac{\partial h}{\partial x_{i}}\right)_{\vec{x}_{0}}\left(x_{i}-x_{i 0}\right)\left(\frac{x_{i 0}}{x_{i}}\right),
\end{aligned}
$$

where $h_{\mathrm{c}}(\vec{x})$ denotes the convex approximation of $h(\vec{x})$ and $\sum_{i}^{(+)}\left(\sum_{i}^{(-)}\right)$represents summation over the variables whose derivatives are positive (negative). The convex approximation is attractive in that it generates the most conservative approximation among all the possible combinations of direct or reciprocal approximations.

If the constraints are underestimated, the design usually ends up in the infeasible region, and many iterations are required to bring it back to the feasible region. Therefore, the design process may either diverge or converge slowly with undesirably large oscillations between the feasible and infeasible regions. The fact that the convex approximation introduces some conservative curvatures in approximate functions accounts for why it may not require control parameters such as move limits. Another attractive feature is that the designer may stop the optimization process at any stage and still obtain a feasible design that is better than the initial design.

The convex approximation is more conservative than either the linear or the reciprocal approximations.
However, this method does not ensure that the approximate constraint is conservative with respect to the real constraint. Therefore, although the convex approximation achieves stable numerical results, the optimum process may either converge slowly if approximate functions are too conservative or continue to oscillate if they are not conservative enough.

\subsection{Two-point exponential approximation and multi-point approximation}

Fadel et al. [5] presented a two-point exponential approximation, which is a linear approximation in terms of intermediate variables $x_{i}^{p_{i}}$. The exponents $p_{i}(i=1,2, \ldots, n)$ are determined by matching function derivatives at the previous data point. It can be expressed in the following form:

$h_{\mathrm{t}}(\vec{x})=h\left(\vec{x}_{0}\right)+\sum_{i=1}^{n}\left(\frac{\partial h}{\partial x_{i}}\right)_{\vec{x}_{0}}\left[\left(\frac{x_{i 1}}{x_{i 0}}\right)^{p_{i}}-1\right]\left(\frac{x_{i 0}}{p_{i}}\right)$,

$p_{i}=1+\frac{\ln \left[\left(\frac{\partial h}{\partial x_{i}}\right)_{\vec{x}_{1}} /\left(\frac{\partial h}{\partial x_{i}}\right)_{\vec{x}_{0}}\right]}{\ln \left(x_{i 1} / x_{i 0}\right)}$,

where $h_{\mathrm{t}}(\vec{x})$ denotes the two-point exponential approximation of $h(\vec{x})$.

Wang and Grandhi [6] developed an improved twopoint function approximation, which not only matches the previous point gradients but also matches its function value. This can be expressed as

$$
\begin{aligned}
h_{\mathrm{ta}}(\vec{x})= & h\left(\vec{x}_{0}\right)+\sum_{i=1}^{n}\left(\frac{\partial h}{\partial x_{i}}\right)_{\vec{x}_{0}}\left[\left(\frac{x_{i 1}}{x_{i 0}}\right)^{p_{i}}-1\right]\left(\frac{x_{i 0}}{p_{i}}\right) \\
& +\frac{1}{2} \varepsilon_{2} \sum_{i=1}^{n}\left(x_{i}^{p_{i}}-x_{i 0}^{p_{i}}\right)^{2},
\end{aligned}
$$

where $h_{\mathrm{ta}}(\vec{x})$ represents the two-point adaptive nonlinear approximation of $h(\vec{x})$ and $\varepsilon_{2}$ is a parameter to be defined.

Differentiating Eq. (7), $n$ equations are obtained by matching the derivatives at the previous point. Another equation is obtained by matching the function value at the previous point. The $n+1$ equations are solved by the numerical iteration method to determine the $p_{i}$ and $\varepsilon_{2}$. However, this approach is limited by the difficulty in obtaining the exponents $p_{i}$. In a related work, Wang and Grandhi [7] developed a multi-point approximation which used Hermite interpolation concepts and the multi-point information generated at the optimization iterations.

Zhang and Fleury [8] presented a modified convex approximation in the following form:

$h_{\mathrm{mc}}(\vec{x})=h_{\mathrm{c}}(\vec{x})+\frac{1}{2} \alpha\left\|\vec{x}_{i}-\vec{x}_{0}\right\|^{2}$, 
where $h_{\mathrm{mc}}(\vec{x})$ denotes the modified convex approximation of $h(\vec{x})$ and $\alpha$ represents the convexity. The value of $\alpha$ can be determined by matching the function value at the previous point. If $\alpha$ is negative, a small positive value will be imposed.

\subsection{Higher-order convex approximation}

When $x_{i}^{2}$ is the intermediate variable if the sensitivity $\left(\partial h / \partial x_{i}\right)_{\vec{x}_{0}}>0$ and $1 / x_{i}^{2}$ is the intermediate variable if $\left(\partial h / \partial x_{i}\right)_{\vec{x}_{0}}<0$, then the convex approximation of order 2 can be written in the following form:

$$
\begin{aligned}
h_{2}(\vec{x})= & h\left(\vec{x}_{0}\right)+\sum_{i}^{(+)} \frac{1}{2}\left(\frac{\partial h}{\partial x_{i}}\right)_{\vec{x}_{0}}\left(\frac{x_{i}^{2}}{x_{i 0}}-x_{i 0}\right) \\
& +\sum_{i}^{(-)} \frac{1}{2}\left(\frac{\partial h}{\partial x_{i}}\right)_{\vec{x}_{0}}\left(\frac{x_{i}^{2}}{x_{i 0}}-x_{i 0}\right)\left(\frac{x_{i 0}}{x_{i}}\right)^{2},
\end{aligned}
$$

where $h_{2}(\vec{x})$ denotes the convex approximation of order 2 .

Similarly, if $x_{i}^{n}$ and $1 / x_{i}^{n}$ are selected as intermediate design variables, the convex approximation of order $n$ can be formulated as the following form:

$$
\begin{aligned}
h_{n}(\vec{x})= & h\left(\vec{x}_{0}\right)+\sum_{i}^{(+)} \frac{1}{n}\left(\frac{\partial h}{\partial x_{i}}\right)_{\vec{x}_{0}}\left[\left(\frac{x_{i}}{x_{i 0}}\right)^{n-1} x_{i}-x_{i 0}\right] \\
& +\sum_{i}^{(-)} \frac{1}{n}\left(\frac{\partial h}{\partial x_{i}}\right)_{\vec{x}_{0}}\left[\left(\frac{x_{i}}{x_{i 0}}\right)^{n-1} x_{i}-x_{i 0}\right]\left(\frac{x_{i 0}}{x_{i}}\right)^{n},
\end{aligned}
$$

where $h_{n}(\vec{x})$ represents the convex approximation of order $n, n$ is an integer and $n \geqslant 2$.

The higher-order, convex approximation is attractive in that it yields more conservative results with the higher convex approximation order. Showing that $h_{n}(\vec{x})<h_{m}(\vec{x})$ for $n<m$ proves this remarkable property. For example, subtracting Eq. (4) from Eq. (9) yields

$$
\begin{aligned}
h_{2}(\vec{x})-h_{\mathrm{c}}(\vec{x})= & \sum_{i}^{(+)} \frac{x_{i 0}}{2}\left(\frac{\partial h}{\partial x_{i}}\right)_{\vec{x}_{0}}\left(\frac{x_{i}}{x_{i 0}}-1\right)^{2} \\
& -\sum_{i}^{(-)} \frac{x_{i 0}}{2}\left(\frac{\partial h}{\partial x_{i}}\right)_{\vec{x}_{0}}\left(\frac{x_{i}}{x_{i 0}}-1\right)^{2} \\
& \times\left(\frac{x_{i 0}}{x_{i}}\right)^{2} \geqslant 0 .
\end{aligned}
$$

For the more general form of higher-order, convex approximation, the real order $r_{i}$ for each $x_{i}$ can be used instead of the integer order $n$. It can be written as

$$
\begin{aligned}
h_{\mathrm{h}}(\vec{x}, \vec{r})= & h\left(\vec{x}_{0}\right)+\sum_{i}^{(+)} \frac{1}{r_{i}}\left(\frac{\partial h}{\partial x_{i}}\right)_{\vec{x}_{0}}\left[\left(\frac{x_{i}}{x_{i 0}}\right)^{r_{i}-1} x_{i}-x_{i 0}\right] \\
& +\sum_{i}^{(-)} \frac{1}{r_{i}}\left(\frac{\partial h}{\partial x_{i}}\right)_{\vec{x}_{0}}\left[\left(\frac{x_{i}}{x_{i 0}}\right)^{r_{i}-1} x_{i}-x_{i 0}\right]\left(\frac{x_{i 0}}{x_{i}}\right)^{r_{i}},
\end{aligned}
$$

where $h_{\mathrm{h}}(\vec{x}, \vec{r})$ denotes the higher-order, convex approximation of $h(\vec{x}), r_{i}$ is a real number and representing the order or degree of convexity of the constraint $h(\vec{x})$ with respect to the design variable $x_{i}$. The value of $r_{i}(i=1,2, \ldots, n)$ varies for each constraint at different iterations. It can be proven that $h_{\mathrm{h}}(\vec{x}, \vec{r})>h_{\mathrm{h}}(\vec{x}, \vec{s})$ if $r_{i}>s_{i}(i=1,2, \ldots, n)$. The proof is given in Appen$\operatorname{dix}$ A.

Many investigations use the three-bar truss, shown in Fig. 1, as an example. This study also uses it to examine the conservative tendency of the proposed method.

The truss is subjected to a concentrated load, and the cross-sectional areas of members 1 and 3 are the same. Two design variables, $x_{1}$ and $x_{2}$ are the cross-sectional areas of members 1 and 2, respectively.

Here, this work examines the approximations of the constraint on the stress in member 1, which stipulates that the stress is less than 20. The theoretical stress of member 1 can be calculated as

$\sigma_{1}=20 \frac{x_{2}+\sqrt{2} x_{1}}{2 x_{1} x_{2}+\sqrt{2} x_{1}^{2}} \leqslant 20$.

Therefore, the actual constraint can be written as

$h(\vec{x})=\frac{x_{2}+\sqrt{2} x_{1}}{2 x_{1} x_{2}+\sqrt{2} x_{1}^{2}}-1 \leqslant 0$.

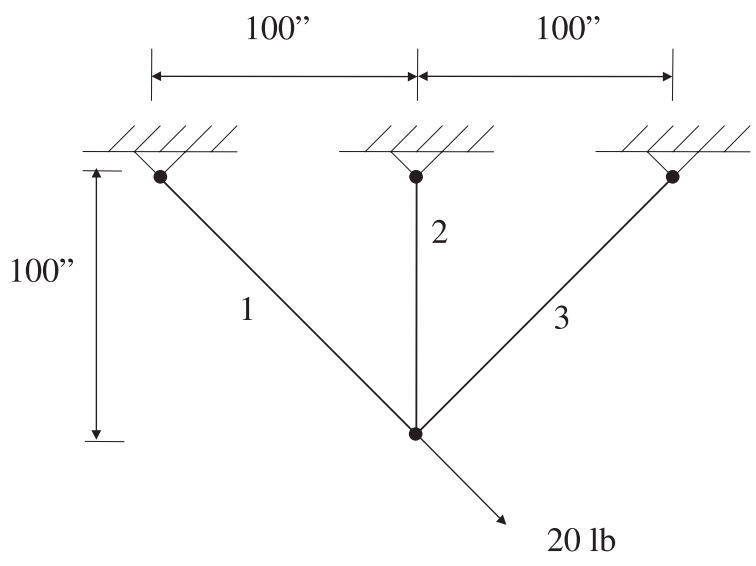

Fig. 1. Three-bar truss. 
Assume that $h(\vec{x})$ is expanded at the point $(1.0,1.0)$. Different kinds of approximations can be constructed as follows:

$$
\begin{aligned}
h_{\mathrm{d}}(\vec{x}) & =8.28-11.714 x_{1}-2.426 x_{2}, \\
h_{\mathrm{r}}(\vec{x}) & =11.714 / x_{1}+2.426 / x_{2}-20, \\
h_{\mathrm{c}}(\vec{x}) & =11.714 / x_{1}+2.426 / x_{2}-20, \\
h_{2}(\vec{x}) & =5.857 / x_{1}^{2}+1.213 / x_{2}^{2}-12.93, \\
h_{\mathrm{h}}(\vec{x},(3.5,3.5)) & =3.347 / x_{1}^{3.5}+0.693 / x_{2}^{3.5}-9.90 .
\end{aligned}
$$

Fig. 2 depicts the different approximations. Notably, the higher the chosen order the more conservative the constraint value. This conservative tendency suggests that if the constraint is not sufficiently conservative, design variable values may oscillate during the optimization steps. In such a case, the approximation order $r_{i}$ in the next iteration should be increased to achieve numerical stability.

\subsection{The self-adjusted convex approximation method}

This study presents an approximation method called self-adjusted convex approximation (SACA). In this method, the order $r_{i}$ for each design variable is adjusted to prevent design variable values oscillating between two consecutive iteration steps. For a lower-order value, the approximate constraint values are probably underesti- mated. Restated, the approximate constraint values are less conservative than the actual constraint values. Under such a condition, the design variable values of the solution of the approximate problem usually oscillates between consecutive iteration steps. Increasing the order is the way to resolve this situation.

In this study, the orders $r_{i}$ for all design variables $x_{i}$ are considered to be the same, i.e.

$r_{i}=r, \quad i=1,2, \ldots, n$.

Therefore, this simplified form of SACA can be written as

$$
\begin{aligned}
h_{\mathrm{a}}(\vec{x}, r)= & h\left(\vec{x}_{0}\right)+\sum_{i}^{(+)} \frac{1}{r}\left(\frac{\partial h}{\partial x_{i}}\right)_{\vec{x}_{0}}\left[\left(\frac{x_{i}}{x_{i 0}}\right)^{r-1} x_{i}-x_{i 0}\right] \\
& +\sum_{i}^{(-)} \frac{1}{r}\left(\frac{\partial h}{\partial x_{i}}\right)_{\vec{x}_{0}}\left[\left(\frac{x_{i}}{x_{i 0}}\right)^{r-1} x_{i}-x_{i 0}\right]\left(\frac{x_{i 0}}{x_{i}}\right)^{r},
\end{aligned}
$$

where $h_{\mathrm{a}}(\vec{x}, r)$ represents the self-adjusted convex approximation of $h(\vec{x})$.

In the $(k-1)$ th iteration, $r^{(k-1)}$ is the order of convex approximation and an approximate problem with respect to $\vec{x}_{k-1}$ can be generated. The optimum solution of this $(k-1)$ th approximate problem is denoted as $\vec{x}_{k}$. With $\vec{x}_{k}$ obtained, the value of $h_{\mathrm{a}}\left(\vec{x}, r^{(k-1)}\right)$ evaluated at $\vec{x}_{k}$ can be obtained and denoted as $h_{\mathrm{a}}\left(\vec{x}_{k}, r^{(k-1)}\right)$.

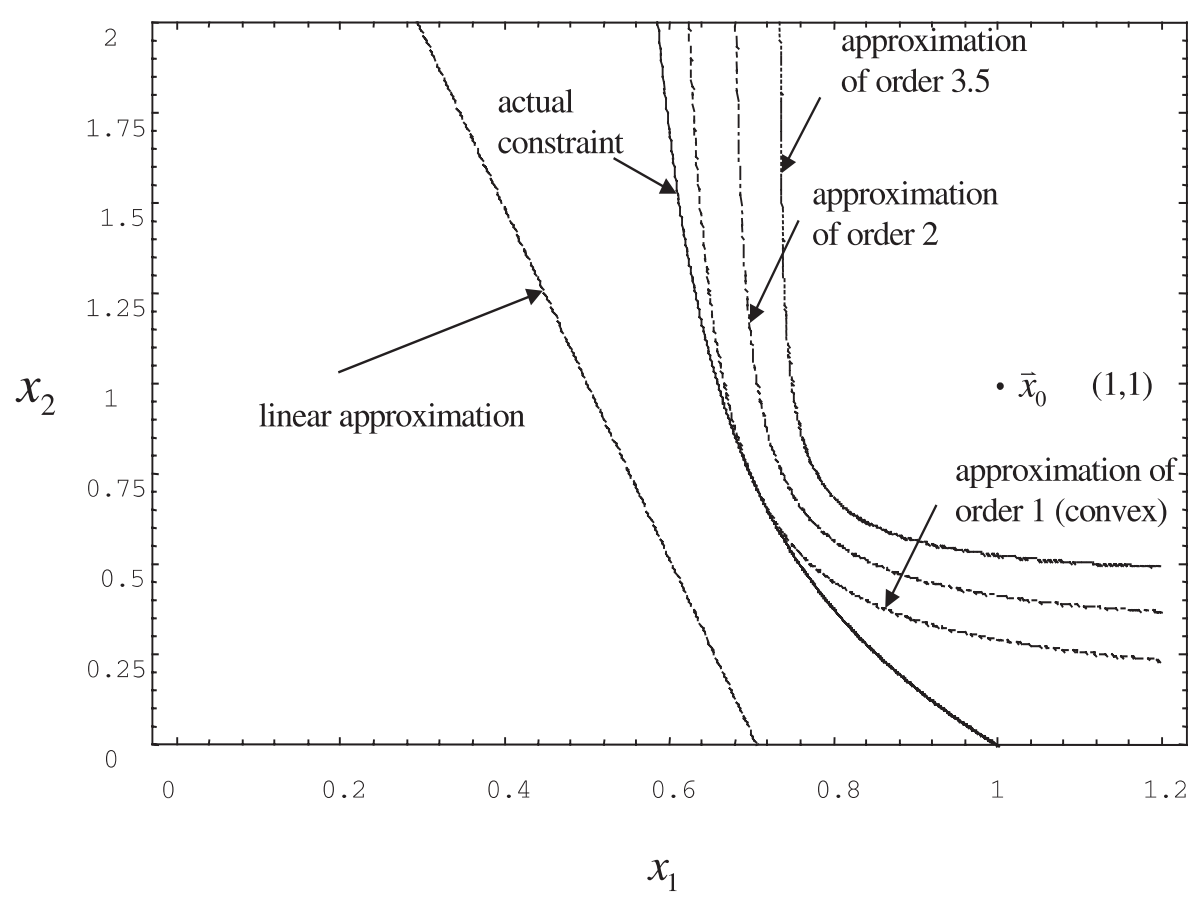

Fig. 2. Comparison of higher-order, convex approximations. 
In the $k$ th iteration, $\vec{x}_{k}$ is the current referent point and the actual constraint value $h\left(\vec{x}_{k}\right)$ can be obtained from the problem analysis procedure. This allows the actual constraint value $h\left(\vec{x}_{k}\right)$ to be compared with the estimated constraint value $h_{\mathrm{a}}\left(\vec{x}_{k}, r^{(k-1)}\right)$. If $h_{\mathrm{a}}\left(\vec{x}_{k}, r^{(k-1)}\right)<h\left(\vec{x}_{k}\right)$, it implies that the approximate function of the $(k-1)$ th iteration is not conservative enough. Restated, it underestimates the actual constraint. Therefore, the order $r^{(k)}$ must be increased to avoid oscillation of design variables and prevent an infeasible design from being obtained. In contrast, if $h_{\mathrm{a}}\left(\vec{x}_{k}, r^{(r-1)}\right)>h\left(\vec{x}_{k}\right)$, it implies that the order is sufficiently conservative; although, it is probably too large and will slow down the convergence. To accelerate the convergence in this case, the order $r^{(k)}$ should be decreased.

The criterion for adjusting the order $r^{(k)}$ is that the approximate function does not underestimate or overestimate the actual constraint value at $\vec{x}_{k}$. That is, $r^{(k)}$ is obtained from solving the equation $h_{\mathrm{a}}\left(\vec{x}_{k}, r\right)=h\left(\vec{x}_{k}\right)$. Substituting this equation into Eq. (16) yields

$$
\begin{aligned}
h\left(\vec{x}_{k-1}\right)+ & \sum_{i}^{(+)} \frac{1}{r}\left(\frac{\partial h}{\partial x_{i}}\right)_{\vec{x}_{k-1}}\left[\left(\frac{x_{i k}}{x_{i(k-1)}}\right)^{r-1} x_{i k}-x_{i(k-1)}\right] \\
& +\sum_{i}^{(-)} \frac{1}{r}\left(\frac{\partial h}{\partial x_{i}}\right)_{\vec{x}_{k-1}}\left[\left(\frac{x_{i k}}{x_{i(k-1)}}\right)^{r-1} x_{i k}-x_{i(k-1)}\right] \\
& \times\left(\frac{x_{i(k-1)}}{x_{i k}}\right)^{r}=h\left(\vec{x}_{k}\right) .
\end{aligned}
$$

The order $r$ is the only unknown in Eq. (17). Because $h_{\mathrm{a}}\left(\vec{x}_{k}, r\right)$ is an increasing function with respect to $r$, as the proof in Appendix A, Eq. (17) can be easily solved by the following steps:

(1) For the increasing function $h_{\mathrm{a}}\left(\vec{x}_{k}, r\right)$, if the upper bound $r_{\mathrm{U}}$ and lower bound $r_{\mathrm{L}}$ of the order $r$ can be determined, the golden section method or other interpolation methods can be used to obtain the proper order $r$ within any given accuracy $\varepsilon$.

(2) $r_{\mathrm{U}}$ and $r_{\mathrm{L}}$ of the order $r$ can be obtained as follows:

(a) If $h_{\mathrm{a}}\left(\vec{x}_{k}, r^{(k-1)}\right)<h\left(\vec{x}_{k}\right)$, the order of the previous iteration $r^{(k-1)}$ is too small to match the actual constraint value at $\vec{x}_{k}$. Therefore, $r^{(k-1)}$ can be set as the lower bound $r_{\mathrm{L}}$ of the order $r$. In this case, the upper bound $r_{\mathrm{U}}$ can be determined by sequentially adding $\Delta r$ to $r_{\mathrm{L}}$ and checking if $h_{\mathrm{a}}\left(\vec{x}_{k}, r\right)>h\left(\vec{x}_{k}\right)$, where $r=r_{\mathrm{L}}+\Delta r$.

(b) If $h_{\mathrm{a}}\left(\vec{x}_{k}, r^{(k-1)}\right)>h\left(\vec{x}_{k}\right)$, the order of the previous iteration $r^{(k-1)}$ can be set as the upper bound $r_{\mathrm{U}}$ of the order $r$. Similarly, the lower bound $r_{\mathrm{L}}$ can be determined by sequentially subtracting $\Delta r$ from $r_{\mathrm{U}}$ and checking if $h_{\mathrm{a}}\left(\vec{x}_{k}, r\right)<h\left(\vec{x}_{k}\right)$, where $r=r_{\mathrm{U}}-\Delta r$.

(3) By the above means, the proper order $r$ of the constraint can be determined. The order $r$ is adopted as the order $r^{(k)}$ of the convex approximation in the $k$ th iteration.

At each iteration, the order for each individual constraint should be solved, respectively. In the following three examples, the time required for a Pentium MMX$200 \mathrm{CPU}$ to determine the order for each constraint is less than $0.01 \mathrm{~s}$. After the approximate constraints are constructed, the approximate structural design problem can be solved by general optimization methods. This study uses the interior penalty method. The total CPU time of one iteration for SACA is about the same as for CONLIN and linear approximation.

The SACA method has three advantages over the two-point approximation methods. First, two-point exponential approximation may not produce an exact $p_{i}$ value due to its negative value in the logarithmic function. However, SACA can always obtain the proper order $r$ because the approximation function is increasing with respect to the order $r$. Second, SACA can easily determine the order $r$ in Eq. (17). In other words, unlike certain two-point approximation methods SACA does not need to utilize the least square method or even to perform another optimization algorithm. Third, the order of SACA is more flexible than the convexity of Eq. (8). The order can be less than 1 or even negative, in other words, the SACA method can be less conservative than CONLIN.

\section{Numerical examples}

\subsection{A three-bar truss}

Consider the three-bar truss in Fig. 1. Table 1 summarizes the design data.

The weight of the truss is considered to be the objective function. The exact solution of this problem can be obtained as [9] $x_{1}=0.788$ in., $x_{2}=0.410$ in., minimum weight $=263.9 \mathrm{lb}$.

Table 2 compares CONLIN and SACA with respect to the structural weight iteration history. Table 3 lists the orders for SACA in the optimization process. It indicates that all the orders are less than 1 . This finding implies that CONLIN is too conservative for this problem. CONLIN takes three more iterations than SACA to converge. Furthermore, comparing the two methods' computation times shows that CONLIN takes $10.11 \mathrm{~s}$ to converge while SACA takes only $5.6 \mathrm{~s}$.

Table 1

Design data for the three-bar truss problem

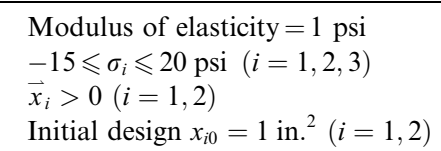


Table 2

Iteration history of the weight for the three-bar truss problem

\begin{tabular}{lll}
\hline Iteration no. & CONLIN & SACA \\
\hline 1 & 267.5 & 267.5 \\
2 & 265.1 & 265.0 \\
3 & 264.4 & 263.9 \\
4 & 264.1 & 263.9 \\
5 & 264.0 & \\
6 & 263.9 & \\
7 & 263.9 & \\
\hline
\end{tabular}

Table 3

The orders for the three-bar truss problem

\begin{tabular}{lccl}
\hline Iteration no. & Constraint 1 & Constraint 2 & Constraint 3 \\
\hline 1 & 0.90 & 0.82 & 0.05 \\
2 & -0.18 & -0.30 & 0.38 \\
3 & -0.28 & -0.44 & 0.54 \\
\hline
\end{tabular}

\subsection{A cantilever beam with a frequency constraint}

This problem involves a cantilever beam with ten cross-sectional area variables $x_{i}(i=1,2, \ldots, 10)$, as shown in Fig. 3, and with the first axial natural frequency constrained to be greater than $410 \mathrm{~Hz}$. Table 4 presents the beam design data and dimensions.

Table 5 compares the iteration history of structural weight for SACA and CONLIN. Some of CONLIN iteration results fall into the infeasible region, in other words, the first natural frequency violates the design constraint. This observation implies that CONLIN is not conservative enough for this example. To illustrate the underestimation of the constraint using convex ap-

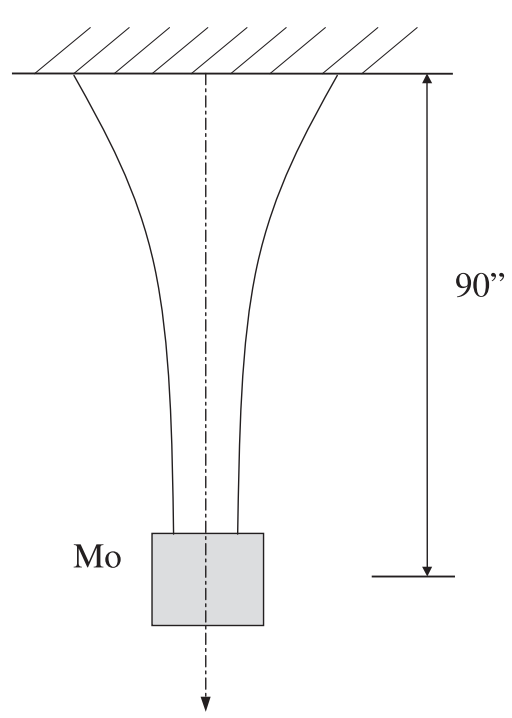

Fig. 3. Cantilever beam with a frequency constraint.
Table 4

Design data for the cantilever beam problem

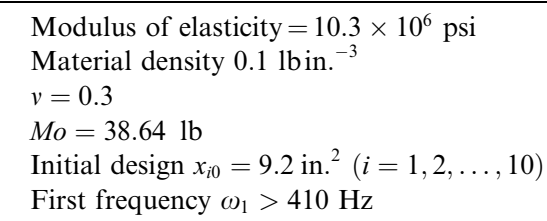

Table 5

Iteration history of the structural weight (lb) for the cantilever beam problem

\begin{tabular}{lll}
\hline Iteration no. & CONLIN & SACA \\
\hline 1 & 71.6 & 71.6 \\
2 & 80.2 & 88.4 \\
3 & 70.5 & 80.1 \\
4 & 77.6 & 80.7 \\
5 & 70.6 & 80.7 \\
6 & 77.6 & \\
7 & 70.6 & \\
8 & 77.6 & \\
9 & 70.6 & \\
10 & 77.6 & \\
\hline
\end{tabular}

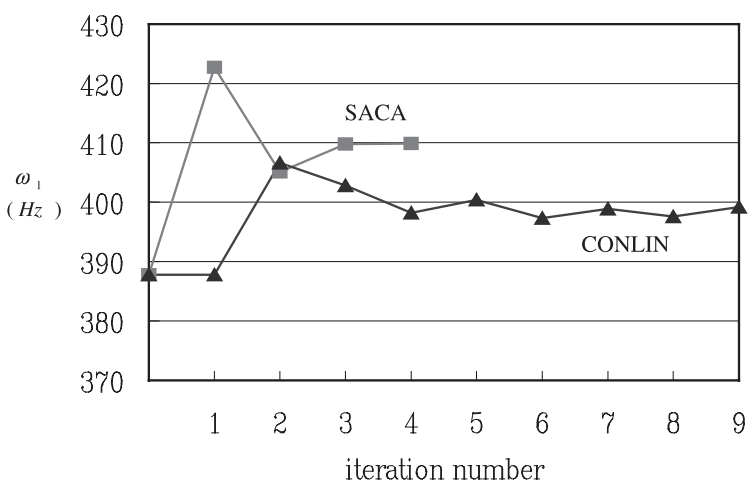

Fig. 4. Natural frequencies in the optimization process.

proximation, Fig. 4 also shows the natural frequency in the iteration history. Table 6 lists the orders of SACA at each iteration step. This table reveals that most orders are greater than 1 , which means CONLIN is not conservative enough.

\subsection{A 10-bar truss with stress constraints}

Fig. 5 shows the minimum weight design of a 10-bar truss [10]. The allowable stress limits are set to $\pm 25 \mathrm{ksi}$ for all members except member 9. For member 9, the allowable stress limit is $\pm 75 \mathrm{ksi}$. The minimum crosssectional area is 0.1 in. $^{2}$, and Table 7 summarizes the design data. 
Table 6

The order for the cantilever beam problem

\begin{tabular}{llllll}
\hline Iteration no. & 1 & 2 & 3 & 4 & 5 \\
Order & 1.00 & 3.90 & 0.32 & 2.44 & 2.61 \\
\hline
\end{tabular}

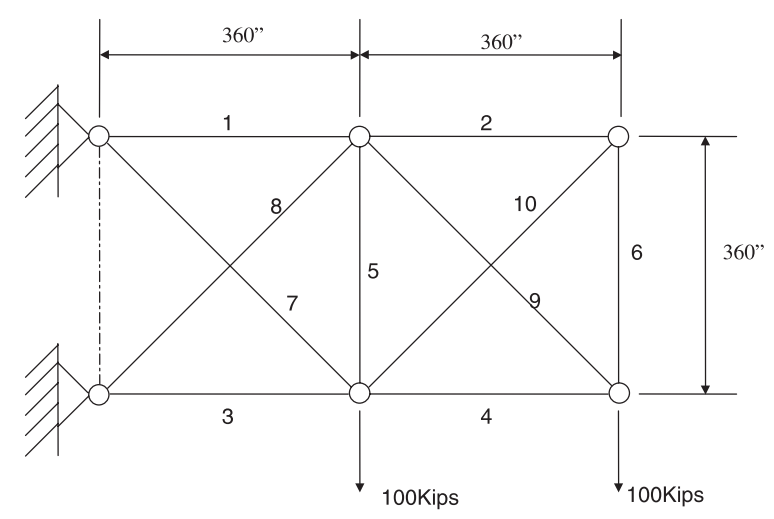

Fig. 5. 10-bar truss with stress constraints.

Table 7

Design data for the 10-bar truss problem

Modulus of elasticity $=1 \times 10^{7} \mathrm{psi}$

Density of the truss material $=0.1 \mathrm{lbin}^{-3}$

$l=360^{\prime \prime}, \mathrm{P}=100 \mathrm{Kips}$

Initial design $x_{i 0}=5$ in. $^{2}(i=1,2, \ldots, 10)$

Table 8

Iteration history of the structural weight (lb) for the 10-bar truss problem

\begin{tabular}{|c|c|c|c|c|c|}
\hline \multirow{2}{*}{$\begin{array}{l}\text { Iteration } \\
\text { no. }\end{array}$} & \multicolumn{4}{|c|}{ Ref. [10] } & \multirow{2}{*}{$\begin{array}{l}\text { This } \\
\text { study, } \\
\text { SACA }\end{array}$} \\
\hline & Linear & $\begin{array}{l}\text { Qua- } \\
\text { dratic }\end{array}$ & $\begin{array}{l}\text { CON- } \\
\text { LIN }\end{array}$ & $\begin{array}{l}\text { Linear } \\
\text { force }\end{array}$ & \\
\hline 1 & 1845 & 2002 & 2361 & 1891 & 1882 \\
\hline 2 & 1637 & 1741 & 1960 & 1688 & 1653 \\
\hline 3 & 1601 & 1650 & 1722 & 1589 & 1550 \\
\hline 4 & 1558 & 1586 & 1641 & 1549 & 1553 \\
\hline 5 & 1531 & 1547 & 1587 & 1526 & 1448 \\
\hline 6 & 1514 & 1525 & 1566 & 1511 & 1491 \\
\hline 7 & 1507 & 1514 & 1555 & 1504 & 1498 \\
\hline 8 & 1502 & 1507 & 1546 & 1501 & 1498 \\
\hline 9 & 1500 & 1503 & 1540 & 1500 & \\
\hline 10 & 1500 & 1501 & 1538 & 1499 & \\
\hline 11 & 1500 & 1500 & 1535 & 1499 & \\
\hline 12 & 1499 & 1499 & 1532 & & \\
\hline
\end{tabular}

Table 8 compares the SACA results with the results in Ref. [10]. In Ref. [10], linear, reciprocal, conservative (CONLIN), quadratic, reciprocal quadratic and linear force approximation was used. The best result was obtained with linear force approximation. Table 8 sum- marizes the iteration history of the structural weight for this problem with Ref. [10] and SACA. This table indicates that SACA obtains the optimum solution with fewer design iterations. Notably, the minimum weight obtained is $1498 \mathrm{lb}$ instead of the $1499 \mathrm{lb}$ obtained in Ref. [10]. This may be attributed to using different finite element numerical algorithms.

\section{Conclusions}

This study presents a method called SACA. The proposed method is a generalization of the conventional convex approximation method. Our results demonstrate that SACA has two merits. First, the order of approximation for each constraint can be easily determined because SACA yields a higher constraint value with a higher order. Second, SACA can adjust the convex order at each redesign iteration to prevent the underestimation or overestimation of design constraints. This improves accuracy and calculation speed by reducing oscillations and speeding up the convergence rate. This study also applies the SACA method to the optimum design processes of three common structural examples, confirming that it yields quite satisfactory results.

\section{Appendix A. The conservative tendency of the higher- order convex approximation}

To prove the conservative tendency of the higherorder convex approximation method, two different set real numbers $\vec{r}$ and $\vec{s}$ are chosen as the order of Eq. (12) and $r_{i}>s_{i}(i=1,2, \ldots, n)$. Then, $h_{\mathrm{h}}(\vec{x}, \vec{r}), h_{\mathrm{h}}(\vec{x}, \vec{s})$ can be expressed as

$$
\begin{aligned}
h_{\mathrm{h}}(\vec{x}, \vec{r})= & h\left(\vec{x}_{0}\right)+\sum_{i}^{(+)} \frac{1}{r_{i}}\left(\frac{\partial h}{\partial x_{i}}\right)_{\vec{x}_{0}}\left[\left(\frac{x_{i}}{x_{i 0}}\right)^{r_{i}-1} x_{i}-x_{i 0}\right] \\
& +\sum_{i}^{(-)} \frac{1}{r_{i}}\left(\frac{\partial h}{\partial x_{i}}\right)_{\vec{x}_{0}}\left[\left(\frac{x_{i}}{x_{i 0}}\right)^{r_{i}-1} x_{i}-x_{i 0}\right]\left(\frac{x_{i 0}}{x_{i}}\right)^{r_{i}}, \\
h_{\mathrm{h}}(\vec{x}, \vec{s})= & h\left(\vec{x}_{0}\right)+\sum_{i}^{(+)} \frac{1}{s_{i}}\left(\frac{\partial h}{\partial x_{i}}\right)_{\vec{x}_{0}}\left[\left(\frac{x_{i}}{x_{i 0}}\right)^{s_{i}-1} x_{i}-x_{i 0}\right] \\
& +\sum_{i}^{(-)} \frac{1}{s_{i}}\left(\frac{\partial h}{\partial x_{i}}\right)_{\vec{x}_{0}}\left[\left(\frac{x_{i}}{x_{i 0}}\right)^{s_{i}-1} x_{i}-x_{i 0}\right]\left(\frac{x_{i 0}}{x_{i}}\right)^{s_{i}} .
\end{aligned}
$$

Subtracting Eq. (A.2) from Eq. (A.1) yields 


$$
\begin{aligned}
h_{\mathrm{h}}(\vec{x}, \vec{r})-h_{\mathrm{h}}(\vec{x}, \vec{s})= & \sum_{i}^{(+)} \alpha_{i} \kappa_{i}\left(\xi_{i}, r_{i}, s_{i}\right) \\
& +\sum_{i}^{(-)} \beta_{i} \lambda_{i}\left(\xi_{i}, r_{i}, s_{i}\right),
\end{aligned}
$$

where

$$
\begin{aligned}
& \xi_{i}=\frac{x_{i}}{x_{i 0}}, \\
& \alpha_{i}=x_{i 0}\left(\frac{\partial h}{\partial x_{i}}\right)_{\mathbf{x}_{0}}>0 \quad \text { if } x_{i 0}>0 \\
& \beta_{i}=x_{i 0}\left(\frac{\partial h}{\partial x_{i}}\right)_{\mathbf{x}_{0}}<0 \quad \text { if } x_{i 0}>0 \\
& \kappa_{i}\left(\xi_{i}, r_{i}, s_{i}\right)=\frac{\xi_{i}^{r_{i}}}{r_{i}}-\frac{\xi_{i}^{s_{i}}}{s_{i}}+\frac{1}{s_{i}}-\frac{1}{r_{i}} \\
& \lambda_{i}\left(\xi_{i}, r_{i}, s_{i}\right)=\frac{\xi_{i}^{-s_{i}}}{s_{i}}-\frac{\xi_{i}^{-r_{i}}}{r_{i}}+\frac{1}{r_{i}}-\frac{1}{s_{i}} .
\end{aligned}
$$

Next, we are going to prove that $\kappa_{i}\left(\xi_{i}, r_{i}, s_{i}\right)>0$ with respect to the variables $\xi_{i}$ for any two given parameters $r_{i}, s_{i} \in \mathbb{R}$ and $r_{i}>s_{i}$. The first and second order derivatives of $\kappa_{i}\left(\xi_{i}, r_{i}, s_{i}\right)$ with respect to $\xi_{i}$ are

$$
\begin{aligned}
\kappa_{i}^{\prime}\left(\xi_{i}, r_{i}, s_{i}\right) & =\frac{\partial \kappa_{i}\left(\xi_{i}, r_{i}, s_{i}\right)}{\partial \xi_{i}}=\xi_{i}^{r_{i}-1}-\xi_{i}^{s_{i}-1}, \\
\kappa_{i}^{\prime \prime}\left(\xi_{i}, r_{i}, s_{i}\right) & =\frac{\partial^{2} \kappa_{i}\left(\xi_{i}, r_{i}, s_{i}\right)}{\partial \xi_{i}^{2}} \\
& =\left(r_{i}-1\right) \xi_{i}^{r_{i}-2}-\left(s_{i}-1\right) \xi_{i}^{s_{i}-2} .
\end{aligned}
$$

The stationary points of $\kappa_{i}\left(\xi_{i}, r_{i}, s_{i}\right)$ can be obtained by solving $\kappa_{i}^{\prime}\left(\xi_{i}, r_{i}, s_{i}\right)=0$,

$\xi_{i}^{r_{i}-1}=\xi_{i}^{s_{i}-1}$

$\xi_{i}^{*}=1$

Substituting $\xi_{i}^{*}=1$ into $\kappa_{i}^{\prime \prime}\left(\xi_{i}, r_{i}, s_{i}\right)$ and $\kappa_{i}\left(\xi_{i}, r_{i}, s_{i}\right)$ gives

$\kappa_{i}^{\prime \prime}\left(\xi_{i}^{*}, r_{i}, s_{i}\right)=r_{i}-s_{i}>0$,

$\kappa_{i}\left(\xi_{i}^{*}, r_{i}, s_{i}\right)=0$

It states that the function $\kappa_{i}\left(\xi_{i}, r_{i}, s_{i}\right)$ has only one minimum point at $\xi_{i}^{*}=1$ and the function value at $\xi_{i}^{*}=1$ is 0 . That is, $\kappa_{i}\left(\xi_{i}, r_{i}, s_{i}\right)>0$

Similarly, it can be proved that

$\lambda_{i}\left(\xi_{i}, r_{i}, s_{i}\right)<0$.

From Eqs. (A.12) and (A.13), Eq. (A.3) can be rewritten as

$$
\begin{aligned}
h_{\mathrm{h}}(\vec{x}, \vec{r})-h_{\mathrm{h}}(\vec{x}, \vec{s})= & \sum_{i}^{(+)} \alpha_{i} \kappa_{i}\left(\xi_{i}, r_{i}, s_{i}\right) \\
& +\sum_{i}^{(-)} \beta_{i} \lambda_{i}\left(\xi_{i}, r_{i}, s_{i}\right)>0 .
\end{aligned}
$$

That is,

$h_{\mathrm{h}}(\vec{x}, \vec{r}) \geqslant h_{\mathrm{h}}(\vec{x}, \vec{s}) \quad$ for $r_{i}>s_{i}(i=1,2, \ldots, n)$.

Therefore, higher-order approximation results in a higher-approximate function value, proving its conservative tendency.

\section{References}

[1] Schmit LA, Farshi B. Some approximation concepts for structural synthesis. AIAA 1974;12(5):692-9.

[2] Storaasli OO, Sobieszczanski-Sobieski J. On the accuracy of the Taylor approximation for structure resizing. AIAA 1974;12:231-3.

[3] Noor AK, Lowder HE. Structural reanalysis via mixed method. Comput Struct 1975;5:9-12.

[4] Fleury C, Braibant V. Structural optimization: a new dual method using mixed variable. Int $\mathbf{J}$ Num Meth Engng 1986;23:409-28.

[5] Fadel GM, Riley MF, Barthelemy JM. Two point exponential approximation method for structural optimization. Struct Optim 1990;2:117-24.

[6] Wang LP, Grandhi RV. Improved two-point function approximations for design optimization. AIAA 1995; 33:1720-7.

[7] Wang LP, Grandhi RV, Canfield RA. Multivariate Hermite approximation for design optimization. Int $\mathbf{J}$ Num Meth Engng 1996;39:787-803.

[8] Zhang WH, Fleury C. A modification of convex approximation methods for structural optimization. Comput Struct 1997;64(1-4):89-95.

[9] Kirsch U. Structural optimization fundamentals and applications. Berlin: Springer; 1993. p. 32-3.

[10] Haftka RT, Gurdal Z. Elements of structural optimization. Dordrecht: Kluwer Academic Publishers, 1992. p. 237-9. 